\title{
Pyrexia after cardiac surgery: Natural history and association with infection
}

Eric Lim, MB, ChB, MRCS ${ }^{\mathrm{a}}$

Reza Motalleb-Zadeh, MB, BChir ${ }^{\mathrm{a}}$

Matthew Wallard, MB, ChB ${ }^{a}$

Nikhil Misra, MB, $\mathrm{ChB}^{\mathrm{a}}$

Enoch Akowuah, MRCS ${ }^{a}$

Ishtiaq Ahmed, MRCS ${ }^{a}$

James C. Halstead, MRCS ${ }^{a}$

Fiona Murphy, $\mathrm{RGN}^{\mathrm{a}}$

Juliet Foweraker, MRCP

Steven Tsui, MD, FRCS ${ }^{a}$

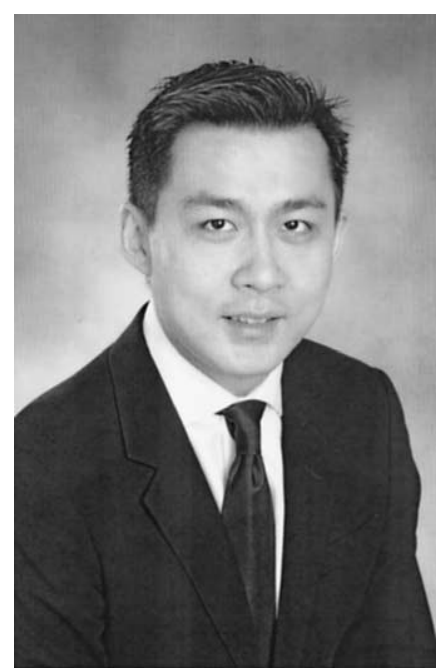

Dr Lim

From the Departments of Cardiothoracic Surgery $^{\mathrm{a}}$ and Microbiology, ${ }^{\mathrm{b}}$ Papworth Hospital, Papworth Everard, Cambridge, United Kingdom.

Received for publication Dec 25, 2002; revisions requested Feb 5, 2003; revisions received Feb 12, 2003; accepted for publication Feb 14, 2003.

Address for reprints: Mr Eric Lim, Department of Cardiothoracic Surgery, Royal Brompton Hospital, Sydney Street, London SW3 6NP, United Kingdom (E-mail: ericlim2@hotmail.com).

J Thorac Cardiovasc Surg 2003;126:1013-7

Copyright (C) 2003 by The American Association for Thoracic Surgery

0022-5223/2003\$30.00+0

doi:10.1016/S0022-5223(03)00363-5
Background: Pyrexia is common after major surgery, and infection is often an important consideration. To investigate the natural history and association with infection, we performed a prospective observational study.

Methods: From November 2000 to January 2001, we studied 219 patients undergoing cardiac surgery screening daily for wound, respiratory, urinary tract, and other infections. Pyrexia was defined as temperature above $37.5^{\circ} \mathrm{C}$.

Results: Of 219 patients, 7 intraoperative deaths occurred and 1 patient was excluded because of preoperative endocarditis, leaving 211. The mean age (SD) was 64 (10) years, consisting of 172 male patients $(81.5 \%)$. The proportion pyrexial on days 1,2 , and 5 was $30.0 \%, 25.8 \%$, and $10.3 \%$, respectively. More patients undergoing urgent or emergency procedures $(17.7 \%$ versus $7.8 \% ; P=.03)$ subsequently developed pyrexia. However, there were no differences in wound infection (3.4\% versus $8.3 \% ; P=.13$ ), positive cultures for respiratory (14.7\% versus $11.4 \%$; $P=.16)$, urinary tract (5.2\% versus $2.0 \% ; P=.09)$, or other infection (8.6\% versus $7.3 \% ; P=.71)$ in patients experiencing postoperative pyrexia compared with those who did not.

Conclusions: Pyrexia is common after cardiac surgery and resolves in the majority of patients by day 5 . Because there is no association between early pyrexia and infection, diagnosis of early postoperative infection by pyrexia alone is insufficient and is better established by clinical assessment with microbiological evidence.

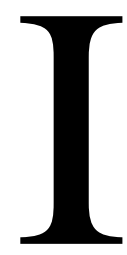

nfection is a primary concern in pyrexial patients after surgery. Pyrexia, however, is so common in the early postoperative course that the clinical utility of this observation to diagnosis infection is diminished. The magnitude of this problem is evident when the clinical significance of pyrexia was deemed to be too uncertain to be included as part of the ASEPSIS score to evaluate wound infection after cardiac surgery. ${ }^{1}$

Pyrexia is also an essential consideration prior to discharge and has emerged as an important cause of delay in fast-track protocols after coronary surgery. ${ }^{2}$ In fact, the implication of low-grade pyrexia is so ambiguous that it is not always considered to be a contraindication to discharge. ${ }^{3}$

To study the natural history and significance of pyrexia after cardiac surgery, we investigated a consecutive series of patients to define the normal range of temperature and assess any correlation between pyrexia and early postoperative infection. 
TABLE 1. Modified ASEPSIS score

\begin{tabular}{|c|c|c|c|c|c|c|}
\hline & & & roportic & Inds af & & \\
\hline & 0 & $<20$ & 21-39 & $40-59$ & $60-79$ & $>80$ \\
\hline Serous exudates & 0 & 1 & 2 & 3 & 4 & 5 \\
\hline Erythema & 0 & 1 & 2 & 3 & 4 & 5 \\
\hline Purulent exudates & 0 & 1 & 4 & 6 & 8 & 10 \\
\hline Separation of deep tissue & 0 & 1 & 4 & 6 & 8 & 10 \\
\hline Additional events within the first 5 days & & & & & & \\
\hline Antibiotics for infection $(\mathrm{n}=10)$ & & & & & & \\
\hline Isolation of bacteria $(n=10)$ & & & & & & \\
\hline Drainage of pus under local anesthesia $(n=5)$ & & & & & & \\
\hline Drainage of pus under general anesthesia $(n=10)$ & & & & & & \\
\hline
\end{tabular}

Sternal wound scores are obtained by the addition of the wound scores on days 1, 3, 4, and 5. Leg wound scores are obtained by the addition of the wound scores on days $2,3,4$, and 5 . Infection was taken as a modified score of more than 15 .

\section{Methods}

We performed a prospective observational study of all patients undergoing cardiac surgery at our institution. Patients with preexisting infection or proceeding to an operation for a complication of infection were excluded.

\section{Study Conduct}

On admission, a data collection booklet was issued to all patients who fulfilled our inclusion criteria; the booklet was completed for preoperative variables before surgery.

Standard prophylactic antibiotics were intravenous flucloxacillin $(1 \mathrm{~g})$ and gentamicin $(2 \mathrm{mg} / \mathrm{kg})$ on induction of anesthesia. For patients who were allergic to penicillin, erythromycin $(1 \mathrm{~g})$ was substituted for flucloxacillin. Operative data were completed by the surgeon or assistant immediately after the procedure.

Postoperatively temperature was measured using a nasopharyngeal probe (DeRoyal, Kells, UK) for the first postoperative night and orally (ThermaDOT, 3M, Neuss, Germany) axillary (BS 691 mercury thermometer, National Health Service, UK) or tympanic (First Temp; Sherwood Medical, Crawley, UK) thereafter. Daily peak temperatures were documented for the purposes of this study. In view of differences in measurement, pyrexia was defined as a maximum temperature of $37.5^{\circ} \mathrm{C}$ or more. Elevated white cell count was defined as $11.0 \times 10^{9}$ cells/ $\mathrm{L}$ or more, the reference range at our institution.

All patients were reviewed daily by a member of the research team and screened for any symptoms or signs suggestive of chest infection (cough and sputum production) or urinary tract infection (frequency or dysuria or hematuria).

Postoperatively, the ward nurses examined both chest and leg wounds. Due to wound-dressing practices and policies of our hospital, we adopted a 4-day ASEPSIS score detailed in Table 1. To promote consistency in wound scoring, all nurses were trained for a month and evaluated during a 2 -week trial period before the commencement of our study. Consistency of scoring was assessed by blind and independent validation between the researchers and observers (nurses). Further education and assessments were performed using pictures of wound infection when discrepancy was noted.

During the course of the study, chest wounds were examined on postoperative days $1,3,4$, and 5 and scored according to our modified ASEPSIS score. Leg wounds were examined and scored similarly on postoperative days 2, 3, 4, and 5. Total 4-day and additional scores were tabulated and wound infection was defined as a modified score of 15 or more. All other infections identified during the course of the hospital stay were documented under the miscellaneous category.

Sputum, urine samples, or wound swabs were ordered for patients with symptoms or signs suggestive of infection. Culture results were obtained and all prescriptions screened to identify any patients who were commenced on antibiotics for infection.

Further investigations included routine white cell counts (on days 1,2, and 4) and plain chest x-rays (on days 1, 3, and 5). Data on white cell counts for days 3 and 5 were included when performed. All chest films were reviewed to document the presence of atelectasis, effusion, and consolidation.

\section{Statistical Methods}

Body temperature was expressed as mean with standard deviation (SD). Baseline variables were compared in the 2 groups using $t$ test for normally distributed variables and Mann-Whitney $U$ test for nonparametric data. Frequencies were expressed as percentages and compared using chi-square analysis.

\section{Results}

From November 6, 2000, to January 20, 2001, we followed up 219 patients who underwent cardiac surgery; 7 deaths occurred and 1 patient was excluded for preoperative endocarditis, leaving 211 patients.

Of 211 patients, 133 underwent coronary artery bypass surgery on bypass, 7 had coronary artery surgery off pump, 39 had heart valve surgery, and 32 patients underwent a combined valve and bypass grafting. The mean age \pm SD was $64 \pm 10$ years old, consisting of 172 male patients $(81.5 \%)$ and 39 female patients $(18.5 \%)$.

In this study, 1016 peak temperatures were recorded from routine temperature measurements in the first 5 days. The method of measurement was tympanic in 642 (64\%), oral in $246(24 \%)$, axillary in $65(6 \%)$, nasopharyngeal in 26 (2\%), and other forms of measurement in $37(4 \%)$. The 


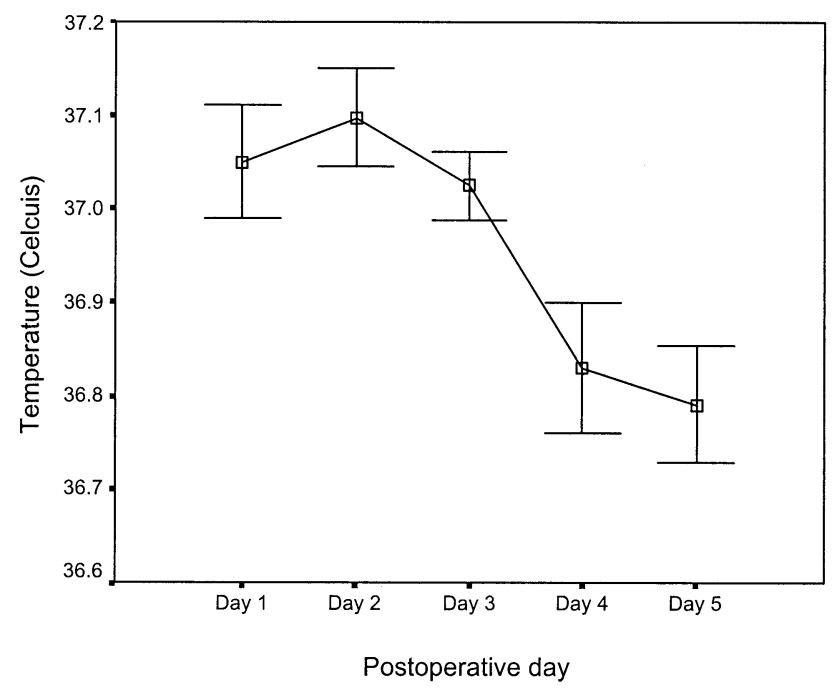

Figure 1. Mean daily peak temperatures in Celsius ( $\pm S E$ ).

overall mean peak temperature on the evening after operation was $37.4 \pm 0.67^{\circ} \mathrm{C}$, and the 5-day temperature profile is summarized in Figure 1. The proportion of patients who were pyrexial on days 1,2 , and 5 were $30.0 \%, 25.8 \%$, and $10.3 \%$, respectively.

The mean white cell count \pm SD on day 1 was $11.6 \pm$ $3.2 \times 10^{9} / \mathrm{L}$ and peaked at $13.0 \pm 3.8 \times 10^{9} / \mathrm{L}$ on day 2 . This declined to $9.8 \pm 2.7 \times 10^{9} / \mathrm{L}$ by day 4 (Figure 2 ). The proportion of patients with an elevated white cell count on day 1 was $53.3 \%$ and peaked at $65.6 \%$ on day 2 , declining to $20.8 \%$ by day 4 .

In our series, $5.7 \%$ (12/211) of patients developed wound infection, and a total of 44 wound swabs were processed. A total of $40.8 \%$ (86/211) of patients developed symptoms of cough productive of sputum, with $9.9 \%(21 / 211)$ of sufficient severity to warrant antibiotic prescription. In all, $13.3 \%(28 / 211)$ of patients had culture-positive respiratory tract pathogens from 50 sputum samples. The frequency of culture-positive urinary tract infection was $3.8 \%(8 / 211)$ from 39 samples, and other miscellaneous culture-positive infections were $8.1 \%(17 / 211)$.

\section{Outcomes Associated with Pyrexia}

Apart from urgency of operation, there were no apparent differences in the baseline characteristics of patients in whom pyrexia subsequently developed during the early postoperative period (Table 2). The difference in median bypass time was not statistically significant between the 2 patient populations. Linear regression analysis, used to test the association between bypass time and peak temperature at 24 hours, did not reveal any significant correlation $(P=$ .36).

A greater proportion of patients who were pyrexial experienced productive cough, and this was accompanied by a

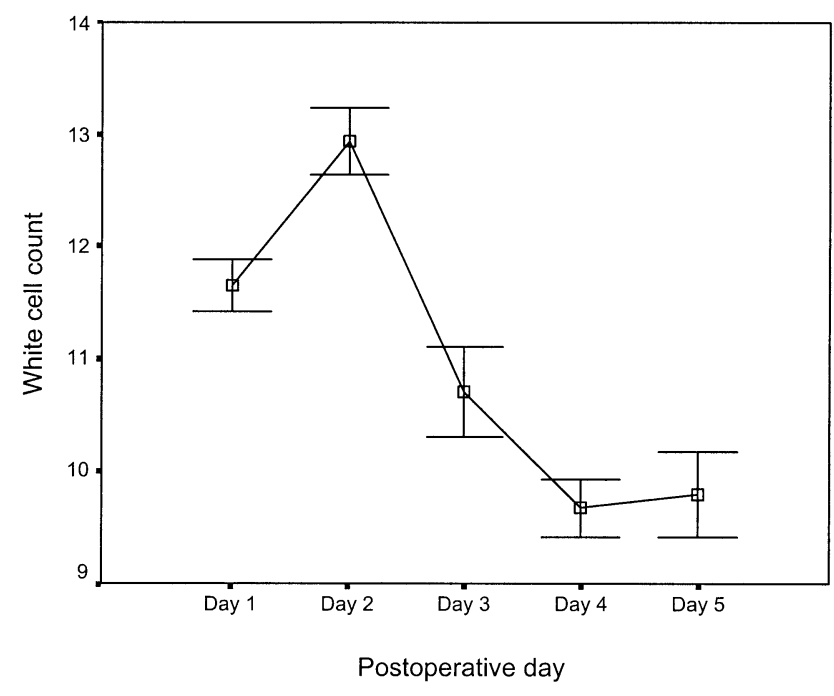

Figure 2. Mean daily white cell counts $\times 10^{9}$ cells/L ( $\pm S E$ ).

similar proportional increase in the frequency of atelectasis (although this did not achieve statistical significance). Interestingly, there was no difference in the frequency of positive sputum culture, other infection, or mean white cell count in patients who developed pyrexia compared with those who did not. At our institution, pyrexia did not influence time to discharge (Table 3).

\section{Discussion}

\section{Natural History}

Pyrexia is a common phenomenon after major surgery. ${ }^{4,5}$ The frequency of postoperative pyrexia after cardiac surgery ranges from $6 \%$ at $38.5^{\circ} \mathrm{C}$ to $30 \%$ at $37.5^{\circ} \mathrm{C}$, depending on the definition of the upper limit of normal body temperature. $^{6}$

Patients tended to have the highest temperature on the first postoperative day and, although pyrexia resolves in the majority, it is evident that prolonged pyrexia and leukocytosis are common, persisting in approximately $10 \%$ of patients at day 5.

\section{Association with Infection}

Our results suggest that the utility of pyrexia or leukocytosis in the diagnosis of infection in the first 5 postoperative days is limited. Patients who developed infection had marginally higher white cell counts that were neither statistically nor clinically significant, with both values within the normal reference range for our laboratories.

We did not find any correlation with either pyrexia or leukocytosis and infection in the early postoperative period, which is consistent with other published work. ${ }^{7}$ However, other groups have shown that the clinical utility of pyrexia and leukocytosis increases from the seventh day, although the specificity remains low at $15 \%{ }^{8}$ 
TABLE 2. Baseline characteristics and risk factors for infection

\begin{tabular}{lccc}
\hline & Pyrexia & No pyrexia & P value \\
\hline Sample size & 115 & 96 & \\
Mean age, years (SD) & $64(11)$ & $65(9)$ & .23 \\
Males, n (\%) & $96(83.5)$ & $76(79.2)$ & .42 \\
Mean BMl, kg/m² (SD) & $27.8(5.5)$ & $27.1(4.7)$ & .34 \\
Diabetes, n (\%) & $13(11.3)$ & $14(14.6)$ & .47 \\
Current smokers, n (\%) & $11(9.6)$ & $12(12.5)$ & .50 \\
COPD, n (\%) & $4(3.5)$ & $4(4.2)$ & .79 \\
Urgent or emergency, n (\%) & $9(7.8)$ & $17(17.7)$ & .03 \\
Median Parsonnet (IQR) & $9(5-13)$ & $10(5-16)$ & .47 \\
Median EuroSCORE (IQR) & $4(2-5)$ & $4(2-6)$ & .57 \\
Median bypass time, minutes (IOR) & $71(60-90)$ & $69(52-88)$ & .09 \\
Median operating time, minutes (IOR) & $180(144-200)$ & $170(144-210)$ & .64 \\
\hline
\end{tabular}

TABLE 3. Pyrexia and postoperative outcome

\begin{tabular}{|c|c|c|c|}
\hline & Pyrexia & No pyrexia & $P$ value \\
\hline \multicolumn{4}{|l|}{ Clinical outcome measures } \\
\hline Cough and sputum, n (\%) & $54(46.9)$ & $32(33.3)$ & .04 \\
\hline Positive sputum culture, $\mathrm{n}(\%)$ & $17(14.7)$ & $11(11.4)$ & .16 \\
\hline Antibiotic prescription, $\mathrm{n}(\%)$ & $18(15.7)$ & $3(3.1)$ & .003 \\
\hline Wound infection, $\mathrm{n}(\%)$ & $4(3.5)$ & $8(8.3)$ & .13 \\
\hline Antibiotic prescription, $\mathrm{n}(\%)$ & $4(3.5)$ & $6(6.2)$ & .42 \\
\hline Urinary tract infection, $\mathrm{n}(\%)$ & $6(5.2)$ & $2(2.0)$ & .09 \\
\hline Antibiotic prescription, $\mathrm{n}(\%)$ & $3(2.6)$ & $1(1.0)$ & .40 \\
\hline Other infections, $\mathrm{n}(\%)$ & $10(8.6)$ & $7(7.3)$ & .71 \\
\hline Median stay, days (IOR) & $9(7$ to 11$)$ & $9(7$ to 13$)$ & .25 \\
\hline \multicolumn{4}{|l|}{ Mean postoperative white cell count } \\
\hline Day $1, \times 10^{9} / \mathrm{L}$ & 11.7 & 11.5 & .76 \\
\hline Day $2, \times 10^{9} / \mathrm{L}$ & 13.2 & 12.6 & .27 \\
\hline Day $4, \times 10^{9} / \mathrm{L}$ & 10.0 & 9.4 & .20 \\
\hline \multicolumn{4}{|l|}{ Radiological features } \\
\hline Atelectasis, $\mathrm{n}(\%)$ & $86(74.8)$ & $61(63.5)$ & .08 \\
\hline Consolidation, n (\%) & $6(5.2)$ & $5(5.2)$ & 1.0 \\
\hline Pleural effusion, $\mathrm{n}(\%)$ & $42(36.5)$ & $38(39.6)$ & .65 \\
\hline
\end{tabular}

\section{Atelectasis}

Pulmonary atelectasis may contribute to the development of postoperative pyrexia. Atelectasis was more frequent in patients with pyrexia; although this did not reach statistical significance, it is consistent with previous work by our institution. Cough with sputum production may be a manifestation of atelectasis, because there were no differences in the frequency of positive sputum culture results to conclude an increase in respiratory tract infection accounted for this disparity. The pattern of decline in the proportion of pyrexial patients from the first postoperative day may be suggestive of the manifestation of systemic inflammation.

\section{The Systemic Inflammatory Response Syndrome}

Disseminated inflammation culminating in the systemic inflammatory response syndrome (SIRS) after cardiac surgery has been attributed to complement activation from contact with foreign surfaces of the pump oxygenator. ${ }^{9}$ Resultant pyrexia after extracorporeal circulation is often attributed to SIRS, but this is difficult to determine as the internationally accepted criteria for $\operatorname{SIRS}^{10}$ is fraught with limitations after cardiac surgery. Heart rate is often kept within defined limits by pacing or pharmacological measures and respiratory rate is altered electively to respiratory performance. With elevated white cell count and pyrexia alone, $14.2 \%$ of patients would satisfy the diagnosis of SIRS after cardiac surgery.

\section{Clinical Implications}

This study has identified deficiencies in the diagnosis of infection and SIRS after cardiac surgery. Traditional indicators of fever and elevated white count alone do not have sufficient discriminating power in the early postoperative 
period to contribute to the diagnosis of infection. Using positive microbiological culture results as the main criteria may miss a significant proportion of patients with culturenegative infections. Using clinical criteria alone is unreliable, particularly for respiratory tract infection, as approximately $40 \%$ of patients experience cough productive of sputum after cardiac surgery. The use of scoring systems for the diagnoses of infections such as wound infection can partially overcome these problems by assigning numerical weighting to each clinical observation. Interestingly, neither pyrexia or white cell count has been included for diagnosis of wound infection in the ASEPSIS scoring system. ${ }^{1}$

\section{Potential Limitations}

The main limitations of studies on infection would be diagnostic criteria. To overcome this problem we have applied a scoring system to assess wound infection that has previously been validated and reported to have low interobserver variation. ${ }^{11}$ In addition, we ensured that training and assessment were undertaken by all nursing staff involved in scoring the wounds. Because there is no consensus on the diagnostic criteria for respiratory tract infection after cardiac surgery, we compared the results of both clinical assessment and microbiology. To minimize bias in the diagnosis of other infections, we used only positive culture results as a hard outcome measure.

Methods used to measure pyrexia were predominantly oral or tympanic. Although we did not standardize method of measurement, we ensured that all tympanic thermometers with electronic methods of measurement were calibrated before the study, and we specified a temperature of $37.5^{\circ} \mathrm{C}$ as a cutoff for pyrexia, which would satisfy both methods of measurement.

All patients received regular paracetamol routinely after surgery, and the peak temperature results from our study should be interpreted in light of the antipyretic effects. However, because this drug is used by most centers for pain relief, the application of our results would be appropriate to most patients.

\section{Conclusion}

Pyrexia and leukocytosis are common phenomena after cardiac surgery that resolve in the majority of patients by postoperative day 5 . Because there is no association between early pyrexia and leukocytosis with early infection, the use of either criterion alone is insufficient to indicate the presence of infection; a diagnosis is better established by clinical assessment supported with microbiological evidence.

We gratefully acknowledge the support of the surgical assistants and intensive care and surgical ward nurses of Papworth Hospital for their contributions to the conduct of this study.

\section{References}

1. Wilson AP, Treasure T, Sturridge MF, Gruneberg RN. A scoring method (ASEPSIS) for postoperative wound infections for use in clinical trials of antibiotic prophylaxis. Lancet. 1986;1:311-3.

2. Loubani M, Mediratta N, Hickey MS, Galinanes M. Early discharge following coronary bypass surgery: is it safe? Eur J Cardiothorac Surg. 2000;18:22-6.

3. Engelman RM. Mechanisms to reduce hospital stays. Ann Thorac Surg. 1996;61:S26-9 (discussion S33-4).

4. Schwandt A, Andrews SJ, Fanning J. Prospective analysis of a fever evaluation algorithm after major gynecologic surgery. Am J Obstet Gynecol. 2001;184:1066-7.

5. Jorgensen FS, Sorensen CG, Kjaergaard J. Postoperative fever after major abdominal surgery. Ann Chir Gynaecol. 1988;77:47-50.

6. Ishikawa S, Ohtaki A, Takahashi T, et al. Management of postoperative fever in cardiovascular surgery. J Cardiovasc Surg (Torino). 1998;39:95-7.

7. Wilson AP, Treasure T, Gruneberg RN, Sturridge MF, Burridge J. Should the temperature chart influence management in cardiac operations? Result of a prospective study in 314 patients. J Thorac Cardiovasc Surg. 1988;96:518-23.

8. Miholic J, Hiertz H, Hudec M, Laczkovics A, Domanig E. Fever, leucocytosis and infection after open heart surgery. A log-linear regression analysis of 115 cases. Thorac Cardiovasc Surg. 1984;32: 45-8.

9. Kirklin JK, Westaby S, Blackstone EH, Kirklin JW, Chenoweth DE, Pacifico AD. Complement and the damaging effects of cardiopulmonary bypass. J Thorac Cardiovasc Surg. 1983;86:845-57.

10. Bone RC, Balk RA, Cerra FB, et al. Definitions for sepsis and organ failure and guidelines for the use of innovative therapies in sepsis. The ACCP/SCCM Consensus Conference Committee. American College of Chest Physicians/Society of Critical Care Medicine. Chest. 1992; 101:1644-55.

11. Wilson AP, Weavill C, Burridge J, Kelsey MC. The use of the wound scoring method "ASEPSIS" in postoperative wound surveillance. $J$ Hosp Infect. 1990;16:297-309. 